\title{
Ideal Convergence of Random Variables
}

\author{
B. Hazarika ${ }^{1}$ and S. A. Mohiuddine ${ }^{2}$ \\ ${ }^{1}$ Department of Mathematics, Rajiv Gandhi University, Rono Hills, Doimukh, Arunachal Pradesh 791 112, India \\ ${ }^{2}$ Department of Mathematics, Faculty of Science, King Abdulaziz University, P.O. Box 80203, Jeddah 21589, Saudi Arabia
}

Correspondence should be addressed to S. A. Mohiuddine; mohiuddine@gmail.com

Received 31 May 2013; Accepted 7 September 2013

Academic Editor: Pankaj Jain

Copyright (C) 2013 B. Hazarika and S. A. Mohiuddine. This is an open access article distributed under the Creative Commons Attribution License, which permits unrestricted use, distribution, and reproduction in any medium, provided the original work is properly cited.

The aim of this paper is to introduce and study the notion of $I$-convergence of random variables via probabilistic norms. Furthermore, we introduce $I$-convergence in $L^{p}$ space and establish some interesting results.

\section{Introduction}

Fast [1] and Steinhaus [2] independently introduced the notion of statistical convergence for sequences of real numbers, which is a generalization of the concept of convergence. The concept of statistical convergence is a very useful functional tool for studying the convergence problems of numerical sequences through the concept of density. Later on, several generalizations and applications of this concept have been presented by various authors (see [3-10] and references therein). Kostyrko et al. [11] presented a generalization of the concept of statistical convergence with the help of ideal $I$ of subsets of the set of natural numbers $\mathbb{N}$ and further studied in [12-16].

Menger [17] presented an interesting and important generalization of the concept of a metric space under the name of statistical metric space by using probability distribution function, which is now called a probabilistic metric space. By using the concept of Menger, Serstnev [18] introduced the concept of probabilistic normed space (for random normed space, see [19]), which is an important generalization of deterministic results of linear normed spaces. Afterward, Alsina et al. [20] presented a new definition of probabilistic normed space which includes the definition of Serstnev as a special case.

The concept of ideal convergence for single and double sequence of real numbers in probabilistic normed space was introduced and studied by Mursaleen and Mohiuddine $[21,22]$. In the recent past, Mursaleen and Alotaibi [23] and Mohiuddine et al. [24] studied the notion of ideal convergence for single and double sequences in random 2normed spaces, respectively. For more detail and related concept, we refer to [25-33] and references therein.

\section{Basic Definitions and Notations}

The notion of statistical convergence depends on the density (asymptotic or natural) of subsets of $\mathbb{N}$. A subset $E$ of $\mathbb{N}$ is said to have natural density $\delta(E)$ if

$$
\delta(E)=\lim _{n \rightarrow \infty} \frac{1}{n}|\{k \leq n: k \in E\}| \text { exists. }
$$

A sequence $x=\left(x_{k}\right)$ is said to be statistically convergent [1] to $\ell$ if for every $\varepsilon>0$

$$
\delta\left(\left\{k \in \mathbb{N}:\left|x_{k}-\ell\right| \geq \varepsilon\right\}\right)=0 .
$$

In this case, we write $S-\lim x=\ell$ or $x_{k} \rightarrow \ell(S)$, and $S$ denotes the set of all statistically convergent sequences.

An ideal is defined as a hereditary and additive family of subsets of a nonempty arbitrary set $X$; here, in our study, it suffices to take $I$ as a family of subsets of $\mathbb{N}$, positive integers; that is, $I \subset 2^{\mathbb{N}}$, such that $\phi \in I, A \cup B \in I$ for each $A, B \in$ $I$, and each subset of an element of $I$ is an element of $I$. A nonempty family of sets $\mathscr{F} \subset 2^{\mathbb{N}}$ is a filter on $\mathbb{N}$ if and only if $\phi \notin \mathscr{F}, A \cap B \in \mathscr{F}$ for each $A, B \in \mathscr{F}$, and any superset of an element of $\mathscr{F}$ is in $\mathscr{F}$. An ideal $I$ is called nontrivial if $I \neq \phi$ and $\mathbb{N} \notin I$. Clearly, $I$ is a nontrivial ideal if and only if 
$\mathscr{F}=\mathscr{F}(I)=\{\mathbb{N}-A: A \in I\}$ is a filter in $\mathbb{N}$, called the filter associated with the ideal $I$. A nontrivial ideal $I$ is called admissible if and only if $\{\{n\}: n \in \mathbb{N}\} \subset I$. A nontrivial ideal $I$ is maximal if there cannot exist any nontrivial ideal $J \neq I$ containing $I$ as a subset. Further details on ideals can be found in Kostyrko et al. (see [11]). Recall that a sequence $x=\left(x_{k}\right)$ of points in $\mathbb{R}$ is said to be $I$-convergent to a real number $\ell$ if $\left\{k \in \mathbb{N}:\left|x_{k}-\ell\right| \geq \varepsilon\right\} \in I$ for every $\varepsilon>0$ (see [11]). In this case, we write $I-\lim x_{k}=\ell$.

Now, we recall some notations and basic definitions that we are going to use in this paper.

We use the notion and terminology of [34]. Thus, $\Delta^{+}$is the space of probability distribution functions $F$ that are left continuous on $\mathbb{R}^{+}=(0,+\infty), F(0)=0$, and $F(+\infty)=$ 1. The space $\Delta^{+}$is partially ordered by the usual pointwise ordering of functions and has both a maximal element $\varepsilon_{0}$ and a minimal element $\varepsilon_{\infty}$; these are given, respectively, by

$$
\begin{gathered}
\epsilon_{0}(t)= \begin{cases}0, & \text { if } t \leq 0 \\
1, & \text { if } t>0,\end{cases} \\
\epsilon_{\infty}(t)= \begin{cases}0, & \text { if } t<+\infty \\
1, & \text { if } t=+\infty\end{cases}
\end{gathered}
$$

There is a natural topology on $\Delta^{+}$that is induced by the modified Lévy metric $d_{L}$ (see, $[34,35]$ ); that is,

$$
d_{L}(F, G)=\inf \{h: \text { both }[F, G ; h] \text { and }[G, F ; h] \text { hold }\}
$$

for all $F, G \in \Delta^{+}$and $h \in(0,1]$, where $[F, G ; h]$ denote the condition

$$
G(t) \leq F(t+h)+h, \quad \text { for } t \in\left(0, \frac{1}{h}\right) .
$$

Convergence with respect to this metric is equivalent to weak convergence of distribution functions, that is $\left(F_{n}\right)$ in $\Delta^{+}$ converges weakly to $F$ in $\Delta^{+}$(written as $F_{n} \stackrel{w}{\rightarrow} F$ ) if and only if $\left(F_{n}(t)\right)$ converges to $F(t)$ at every point of continuity of the limit function $F$. Consequently, we have

$$
\begin{gathered}
F_{n} \stackrel{w}{\longrightarrow} F \quad \text { iff } d_{L}\left(F_{n}, F\right) \longrightarrow 0, \\
F(x)>1-x \quad \text { iff } d_{L}\left(F, \varepsilon_{0}\right)<x \text { for every } x>0 .
\end{gathered}
$$

Moreover, the metric space $\left(\Delta^{+}, d_{L}\right)$ is compact.

Definition 1. A triangular norm (or briefly, t-norm) is a binary operation $T:[0,1] \times[0,1] \rightarrow[0,1]$ that satisfies the following conditions (see [36]):

(TN1) $T(s, t)=T(t, s)$ for all $s, t \in[0,1]$ ( $T$ is commutative),

(TN2) $T(T(s, t), u)=T(s, T(t, u))$ for all $s, t, u \in[0,1](T$ is associative),

(TN3) $T(s, t) \leq T\left(s^{\prime}, t\right)$ for all $t \in[0,1]$ whenever $s \leq s^{\prime}(T$ is nondecreasing),

(TN4) $T(1, t)=t$ for every $t \in[0,1]$ ( $T$ satisfies the boundary condition).
$T^{*}$ is a continuous $t$-conorm, namely, a continuous binary operation on $[0,1]$ that is related to a continuous $t$-norm through $T^{*}(s, t)=1-T(1-s, 1-t)$.

Notice that by virtue of its commutativity, any $t$-norm $T$ is nondecreasing in each place. Some examples of $t$-norms $T$ and its $t$-conorms $T^{*}$ are $W(x, y)=\max \{x+y-1,0\}$, $M(x, y)=\min \{x, y\}$, and $\Pi(x, y)=x \cdot y$ and $W^{*}(x, y)=$ $\min \{x+y, 1\}, M^{*}(x, y)=\max \{x, y\}$, and $\Pi^{*}(x, y)=x+y-$ $x \cdot y$.

Definition 2. A triangle function is a binary operation on $\Delta^{+}$, namely, a function $\tau: \Delta^{+} \times \Delta^{+} \rightarrow \Delta^{+}$that is associative, commutative, and nondecreasing and which has $\varepsilon_{0}$ as unit; that is, for all $F, G, H, K \in \Delta^{+}$, one has:
(1) $\tau\left(F, \epsilon_{0}\right)=F$;
(2) $\tau(F, G)=\tau(G, F)$;
(3) $\tau(F, G) \leq \tau(H, K)$ whenever $F \leq H, G \leq K$;
(4) $\tau(\tau(F, G), H)=\tau(F, \tau(G, H))$.

Particular and relevant triangle functions are the functions $\tau_{T}, \tau_{T^{*}}$ and those of the form $\Pi_{T}$ which, for any continuous $t$-norm $T$ and any $x>0$, are given by

$$
\begin{gathered}
\tau_{T}(F, G)(x)=\sup \{T(F(u), G(v)): u+v=x\}, \\
\tau_{T^{*}}(F, G)(x)=\inf \left\{T^{*}(F(u), G(v)): u+v=x\right\}, \\
\Pi_{T}(F, G)(x)=T(F(x), G(x)) .
\end{gathered}
$$

Definition 3. A probabilistic normed space (or briefly, PN space) is a quadruple $\left(X, v, \tau, \tau^{*}\right)$, where $X$ is a real linear space, $\tau$ and $\tau^{*}$ are continuous triangle functions such that $\tau \leq \tau^{*}$, and the mapping $\nu: X \rightarrow \Delta^{+}$called the probabilistic norm, for all $p$ and $q$ in $X$, satisfies the following conditions:

(PN1) $v_{p}=\epsilon_{0}$ if and only if $p=\theta(\theta$ is the null vector in $X)$; (PN2) for all $p \in X, v_{-p}=v_{p}$;

(PN3) $v_{p+q} \geq \tau\left(v_{p}, v_{q}\right)$

(PN4) for all $a \in[0,1], \nu_{p} \leq \tau^{*}\left(\nu_{a p}, \nu_{(1-a) p}\right)$.

If a PN space $\left(X, \nu, \tau, \tau^{*}\right)$ satisfies the following condition:

( $\check{S})$ for all $p \in X$, for all $\lambda \in \mathbb{R} \backslash\{0\}$, for all $t>0$, $v_{\lambda p}(t)=v_{p}(t /|\lambda|)$

then it is called a Šerstnev space; the condition $(\check{S})$ implies that the best-possible selection for $\tau^{*}$ is $\tau^{*}=\tau_{M}$, which satisfies a stricter version of (PN4); namely,

$$
\forall a \in[0,1], \quad \nu_{p}=\tau_{M}\left(\nu_{a p}, \nu_{(1-a) p}\right) .
$$

A Šerstnev space is denoted by $(X, v, \tau)$, since the role of $\tau^{*}$ is placed by a fixed triangle function $\tau_{M}$, which satisfies (PN2).

A PN space $X$ is endowed with the strong topology (briefly $\mathcal{S}$-topology) generated by the strong neighborhood system $\left\{\mathcal{N}_{\theta}(\lambda): \lambda>0\right\}$, where

$$
\mathcal{N}_{\theta}(\lambda)=\left\{p \in X: d_{L}\left(v_{p}, \varepsilon_{0}\right)<\lambda\right\}
$$


determines a first countable and Hausdorff topology on $X$ (see [34]), and it is metrizable.

The following lemma is an immediate consequence of the definition of neighborhood of zero and (7).

Lemma 4. In a PN space $\left(X, \nu, \tau, \tau^{*}\right)$, for each $p \in X$, one has

$$
\nu_{p}(\lambda)>1-\lambda \Longleftrightarrow p \in \mathcal{N}_{\theta}(\lambda) .
$$

A sequence $\left(p_{n}\right)$ of elements in $X$ converges to $\theta$, the null element of $X$, in the strong topology (briefly $\mathcal{S}$-topology) (written $p_{n} \rightarrow \theta$ ) if and only if

$$
\lim _{n \rightarrow \infty} d_{L}\left(v_{p_{n}}, \varepsilon_{0}\right)=0
$$

That is, for every $\lambda>0$, there is an integer $m=m(\lambda) \in \mathbb{N}$ such that $d_{L}\left(\nu_{p_{n}}, \theta\right)<\lambda$ for all $n \geq m$, where $d_{L}$ is defined in (4). In terms of neighborhood, we have $p_{n} \rightarrow \theta$ provided that for any $\lambda>0$ there is an $N(\lambda) \in \mathbb{N}$ such that $p_{n} \in \mathcal{N}_{\theta}(\lambda)$ (i.e., $\left.v_{p_{n}}(\lambda)>1-\lambda\right)$ whenever $n \geq N$. In this case, we write $p_{k} \stackrel{\mathcal{S}}{\rightarrow} \theta$ or $\mathcal{S}-\lim _{k} p_{k}=\theta$. Thus, the $\mathcal{S}$-topology can be completely specified by means of $\mathcal{S}$-convergence of sequences.

A sequence $\left(p_{k}\right)$ is said to be $\mathcal{S}$-Cauchy if for any $\lambda>0$, there exists an integer $N(\lambda) \in \mathbb{N}$ such that $p_{k}-p_{n} \in \mathcal{N}_{\theta}(\lambda)$ whenever $k, n \geq N(\lambda)$.

Lemma 5 (see [37]). For any $\alpha \in \mathbb{R}$, any $p \in X$, and any $\varepsilon>0$, there exists a $\lambda>0$ such that

$$
\alpha p \in \mathcal{N}_{\theta}(\varepsilon) \quad \text { whenever } p \in \mathcal{N}_{\theta}(\lambda) \text {. }
$$

Lemma 6 (see [37]). If $0 \leq \alpha<1$, then for any $p \in X$

$$
v_{\alpha p} \geq v_{p}
$$

Lemma 7 (see [37]). For any $\alpha \in \mathbb{N}$, any $p \in X$, and any $t>0$, there is a $\lambda>0$ such that

$$
d_{L}\left(\nu_{\alpha p}, \varepsilon_{0}\right)<t \quad \text { whenever } d_{L}\left(\nu_{p}, \varepsilon_{0}\right)<\lambda
$$

We observe that, in view of Lemma 4 and (PN3), we have the following lemma.

Lemma 8. Let $\left(X, v, \tau, \tau^{*}\right)$ be a PN space. For all $p, q, r \in X$,

$$
d_{L}\left(\nu_{p-r}, \varepsilon_{0}\right) \leq d_{L}\left(\tau\left(\nu_{p-q}, v_{q-r}\right), \varepsilon_{0}\right) .
$$

An important class of PN spaces is that of $E$-normed spaces (see [38]). Let $(\Omega, \mathscr{A}, P)$ be a probability space, $(X,\|\cdot\|)$ a normed space, and $S$ a linear space of $X$-valued random variables (possibly, the entire space). For every $p \in S$ and for every $\lambda \in \mathbb{R}$, let $\nu: S \rightarrow \Delta^{+}$be defined by

$$
\nu_{\lambda}:=P(\{\omega \in \Omega:\|p(\omega)\|<\lambda\}) ;
$$

then $(S, v)$ is an $E$-normed space (briefly, EN space) with the base $(\Omega, \mathscr{A}, P)$ and target $(X,\|\cdot\|)$.
Example 9. Let $L^{0}=L^{0}(\Omega, \mathscr{A}, P)$, the linear space of (equivalence classes of) random variable $f: \Omega \rightarrow \mathbb{R}$. Let $\nu: S \rightarrow \Delta^{+}$be defined, for every $f \in L^{0}$ and for every $\lambda \in \mathbb{R}$, by

$$
v_{f}(\lambda)=P(\{\omega \in \Omega:|f(\omega)|<\lambda\})
$$

Then, the couple $\left(L^{0}, v\right)$ is an EN space. It is a $\mathrm{PN}$ space under the triangle function $\tau_{W}$ and $\tau_{M}$ (see [34]).

\section{Ideal Convergence of Random Variables}

Throughout the paper, we denote $I$ as an admissible ideal of subsets of $\mathbb{N}$, unless otherwise stated. In this section, we begin with the definition of ideal convergence of probability distribution functions.

Definition 10. Let $I \subset 2^{\mathbb{N}}$, and let $\left(\Delta^{+}, d_{L}\right)$ be a Lévy metric space. A sequence $\left(F_{k}\right)$ in $\Delta^{+}$is said to be I-convergent (weakly) to $F \in \Delta^{+}$if and only if for every $\lambda>0$, the set

$$
\left\{k \in \mathbb{N}: d_{L}\left(F_{k}, F\right) \geq \lambda\right\} \in I,
$$

or

$$
\left\{k \in \mathbb{N}: d_{L}\left(F_{k}, F\right)<\lambda\right\} \in \mathscr{F} .
$$

In this case, we write $F_{k} \stackrel{\mathscr{W} I}{\longrightarrow} F$ or $\mathscr{W} I-\lim F_{k}=F$.

By (7) and (19), the following lemma can be easily verified.

Lemma 11. Let $\left(\Delta^{+}, d_{L}\right)$ be a Lévy metric space and $\left(F_{k}\right)$ a sequence in $\Delta^{+}$. Then, for every $\lambda>0$, the following statements are equivalent:
(i) $F_{k} \stackrel{\mathscr{W} I}{\longrightarrow} \varepsilon_{0}$,
(ii) $\left\{k \in \mathbb{N}: d_{L}\left(F_{k}, \varepsilon_{0}\right)<\lambda\right\} \in \mathscr{F}$,
(iii) $\left\{k \in \mathbb{N}: F_{k}(\lambda)>1-\lambda\right\} \in \mathscr{F}$,
(iv) $\mathscr{W} I-\lim _{k} d_{L}\left(F_{k}, \varepsilon_{0}\right)=0$.

Definition 12. Let $\left(X, v, \tau, \tau^{*}\right)$ be a PN space. A sequence $\left(p_{k}\right)$ in $X$ is said to be $I$-convergent to $\theta$ in the strong topology (or strong-I-convergent) if and only if for every $\lambda>0$, the set

$$
\left\{k \in \mathbb{N}: d_{L}\left(v_{p_{k}}, \varepsilon_{0}\right) \geq \lambda\right\} \in I,
$$

or

$$
\left\{k \in \mathbb{N}: d_{L}\left(\nu_{p_{k}}, \varepsilon_{0}\right)<\lambda\right\} \in \mathscr{F}
$$

In this case, we write $p_{k} \stackrel{\mathcal{S} I}{\longrightarrow} \theta$ or $\delta I-\lim p_{k}=\theta$, where $\theta$ is called the $\delta I$-limit of $\left(p_{k}\right)$. In terms of neighborhoods, we have

$$
p_{k} \stackrel{\mathcal{S I}}{\longrightarrow} \theta \quad \text { iff }\left\{k \in \mathbb{N}: p_{k} \notin \mathcal{N}_{\theta}(\lambda)\right\} \in I .
$$

The following lemma is an immediate consequence of the above definition. 
Lemma 13. Let $\left(X, v, \tau, \tau^{*}\right)$ be a PN space and $\left(p_{k}\right)$ a sequence in $X$. Then, for every $\lambda>0$, the following statements are equivalent:

(i) $p_{k} \stackrel{\mathcal{S I}}{\longrightarrow} \theta$,

(ii) $\left\{k \in \mathbb{N}: p_{k} \notin \mathcal{N}_{\theta}(\lambda)\right\} \in I$,

(iii) $\left\{k \in \mathbb{N}: d_{L}\left(v_{p_{k}}, \varepsilon_{0}\right) \geq \lambda\right\} \in I$.

Theorem 14. Let $\left(X, v, \tau, \tau^{*}\right)$ be a PN space, and if a sequence $\left(p_{k}\right)$ in $X$ is $\delta I$-convergent, then $\mathcal{S} I-\lim p_{k}$ is unique.

Proof. Suppose that $\delta I-\lim p_{k}=p$ and $\delta I-\lim p_{k}=q$ with $p \neq q$. Then, for $\lambda>0$, define the following sets:

$$
\begin{aligned}
& K_{1}(\lambda)=\left\{k \in \mathbb{N}: p_{k}-p \notin \mathcal{N}_{\theta}(\lambda)\right\}, \\
& K_{2}(\lambda)=\left\{k \in \mathbb{N}: p_{k}-q \notin \mathcal{N}_{\theta}(\lambda)\right\} .
\end{aligned}
$$

Since $\delta I-\lim p_{k}=p$, using Lemma 13 , we have $K_{1}(\lambda) \in$ $I$. Also, using $\mathscr{S} I-\lim p_{k}=q$, we get $K_{2}(\lambda) \in I$. Let

$$
K(\lambda)=K_{1}(\lambda) \cup K_{2}(\lambda) .
$$

Then, $K(\lambda) \in I$ for all $\lambda>0$. This implies that its complement $K^{c}(\lambda)$ is a nonempty set in $\mathscr{F}$ for all $\lambda>0$. Now, if $k \in K^{c}(\lambda)$, then $p_{k}-p \in \mathcal{N}_{\theta}(\lambda)$. Let $d_{L}\left(v_{p_{k}-p}, \varepsilon_{0}\right)=\alpha$. Then, $\lambda-\alpha>$ 0 , and the uniform continuity of $\tau$ implies that there exists a $\lambda_{1}>0$ such that

$$
d_{L}\left(\tau\left(v_{p_{k}-p}, G\right), v_{p_{k}-p}\right)<\lambda-\alpha
$$

whenever $d_{L}\left(G, \varepsilon_{0}\right)<\lambda_{1}$. Now, let $p_{k}-q \in \mathcal{N}_{\theta}\left(\lambda_{1}\right)$, and then $d_{L}\left(v_{p_{k}-q}, \varepsilon_{0}\right)<\lambda_{1}$. Thus, by (16), we have

$$
\begin{aligned}
d_{L}\left(v_{p-q}, \varepsilon_{0}\right) & \leq d_{L}\left(\tau\left(v_{p_{k}-p}, v_{p_{k}-q}\right), \varepsilon_{0}\right) \\
& \leq d_{L}\left(\tau\left(v_{p_{k}-p}, v_{p_{n}-q}\right), v_{p_{k}-p}\right)+d_{L}\left(v_{p_{k}-p}, \varepsilon_{0}\right) \\
& <\lambda-\alpha+\alpha=\lambda .
\end{aligned}
$$

Hence, $p-q \in \mathcal{N}_{\theta}(\lambda)$. Since $\lambda$ is arbitrary, we get $v_{p-q}=$ $\varepsilon_{0}$, which yields $p-q=0$; that is, $p=q$. Thus, this completes the proof.

The next theorem gives the algebraic characterization of $\delta I$-convergence in PN space.

Theorem 15. Let $\left(X, v, \tau, \tau^{*}\right)$ be a PN space and $\left(p_{k}\right)$ and $\left(q_{k}\right)$ two sequences in $X$.

(a) If $\delta I-\lim p_{k}=p$ and $c \in \mathbb{R}$, then $\delta I-\lim c p_{k}=c p$.

(b) If $\delta I-\lim p_{k}=p$ and $\delta I-\lim q_{k}=q$, then $\delta I-$ $\lim \left(p_{k} \pm q_{k}\right)=p \pm q$.

Proof. (a) Let $c \in \mathbb{R}$, and let $\left(p_{n}\right)$ be a sequence in $X$ such that $\delta I-\lim p_{k}=p$. Then, from (15), we have

$$
\left\{k \in \mathbb{N}: d_{L}\left(\nu_{p_{k}-p}, \varepsilon_{0}\right)<\lambda\right\} \subset\left\{k \in \mathbb{N}: d_{L}\left(\nu_{c\left(p_{k}-p\right)}, \varepsilon_{0}\right)<t\right\}
$$

for any $t>0$. Since $\delta I-\lim p_{k}=p$, we have

$$
\left\{k \in \mathbb{N}: d_{L}\left(v_{p_{k}-p}, \varepsilon_{0}\right)<\lambda\right\} \in \mathscr{F} .
$$

Thus, we have for each $t>0$

$$
\left\{k \in \mathbb{N}: d_{L}\left(v_{c\left(p_{k}-p\right)}, \varepsilon_{0}\right)<t\right\} \in \mathscr{F} .
$$

This shows that $\delta I-\lim c p_{k}=c p$.

(b) Let $\left(p_{k}\right)$ and $\left(q_{k}\right)$ be two sequences in $X$ such that $\delta I-$ $\lim p_{k}=p$ and $\delta I-\lim q_{k}=q$. Then, for $\lambda>0$, define the following sets:

$$
\begin{aligned}
& A(\lambda)=\left\{k \in \mathbb{N}: p_{k}-p \notin \mathcal{N}_{\theta}(\lambda)\right\}, \\
& B(\lambda)=\left\{k \in \mathbb{N}: q_{k}-q \notin \mathcal{N}_{\theta}(\lambda)\right\} .
\end{aligned}
$$

Now, we can write

$$
\nu_{\left(p_{k}+q_{k}\right)-(p+q)} \geq \tau\left(v_{p_{k}-p}, v_{q_{k}-q}\right),
$$

and hence,

$$
\begin{array}{r}
d_{L}\left(v_{\left(p_{k}+q_{k}\right)-(p+q)}, \varepsilon_{0}\right) \leq d_{L}\left(\tau\left(v_{p_{k}-p}, v_{q_{k}-q}\right), \varepsilon_{0}\right) \\
\text { for every } k \in \mathbb{N} .
\end{array}
$$

By uniform continuity of $\tau$, we can say that for any $t>0$ there exists a $\lambda>0$ such that $d_{L}\left(\tau(F, G), \varepsilon_{0}\right)<t$ whenever $d_{L}\left(F, \varepsilon_{0}\right)<\lambda$ and $d_{L}\left(G, \varepsilon_{0}\right)<\lambda$, where $F, G \in \Delta^{+}$. Now, let $t>0$. Then, we can find a $\lambda>0$ such that

$$
d_{L}\left(\tau\left(v_{p_{k}-p}, v_{q_{k}-q}\right), \varepsilon_{0}\right)<t
$$

that is,

$$
d_{L}\left(\nu_{\left(p_{k}+q_{k}\right)-(p+q)}, \varepsilon_{0}\right)<t
$$

whenever $p_{k}-p \in \mathcal{N}_{\theta}(\lambda)$ and $q_{k}-q \in \mathcal{N}_{\theta}(\lambda)$, that is, $d_{L}\left(\nu_{p_{k}-p}, \varepsilon_{0}\right)<\lambda$ and $d_{L}\left(\nu_{q_{k}-q}, \varepsilon_{0}\right)<\lambda$. Thus, we have

$$
\begin{aligned}
& \left\{k \in \mathbb{N}: d_{L}\left(\nu_{\left(p_{k}+q_{k}\right)-(p+q)}, \varepsilon_{0}\right) \geq t\right\} \\
& \quad \subseteq\left\{k \in \mathbb{N}: p_{k}-p \notin \mathcal{N}_{\theta}(\lambda)\right\} \cup\left\{k \in \mathbb{N}: q_{k}-q \notin \mathcal{N}_{\theta}(\lambda)\right\}
\end{aligned}
$$

for each $t>0$.

Then, for each $t>0$, we have

$$
\left\{k \in \mathbb{N}: d_{L}\left(\nu_{\left(p_{k}+q_{k}\right)-(p+q)}, \varepsilon_{0}\right) \geq t\right\} \subseteq A(\lambda) \cup B(\lambda) .
$$

Since $I$ is admissible, from (37), we have

$$
\left\{k \in \mathbb{N}: d_{L}\left(\nu_{\left(p_{k}+q_{k}\right)-(p+q)}, \varepsilon_{0}\right) \geq t\right\} \in I \quad \text { for each } t>0 \text {. }
$$

Hence, $\delta I-\lim \left(p_{k}+q_{k}\right)=p+q$.

Similarly, we can show that $\mathcal{S} I-\lim \left(p_{k}-q_{k}\right)=p-q$.

Theorem 16. Let $\left(X, v, \tau, \tau^{*}\right)$ be a PN space, and let $\left(p_{k}\right)$ be a sequence in $X$. If $\mathcal{S}-\lim p_{k}=p$, then $\mathcal{S} I-\lim p_{k}=p$. 
Proof. Let $\mathcal{S}-\lim p_{k}=p$; then for every $\lambda>0$ there exists an integer $N=N(\lambda) \in \mathbb{N}$ such that

$$
p_{k}-p \in \mathcal{N}_{\theta}(\lambda) \quad \text { whenever } k \geq N(\lambda) .
$$

Therefore, the set

$$
B(\lambda)=\left\{k \in \mathbb{N}: p_{k}-p \notin \mathcal{N}_{\theta}(\lambda)\right\} \subseteq\{1,2, \ldots, N-1\} .
$$

But, with $I$ being admissible, we have $B(\lambda) \in I$. Hence, $\delta I-$ $\lim p_{k}=p$.

\section{Theorem 17. Sequential method SI is regular.}

Proof. The proof follows from the fact that $I$ is admissible and from Theorem 16.

Theorem 18. Let $\left(X, v, \tau, \tau^{*}\right)$ be a PN space. A sequence $\left(p_{k}\right)$ in $X$ is $\delta I$-convergent to $\theta$ if and only if there exists a subset $K=\{i\} \subset \mathbb{N}$ such that $K \in \mathscr{F}$ and $\mathcal{S}-\lim _{k \in K, k \rightarrow \infty} p_{k}=\theta$.

Proof. Suppose that $\delta I-\lim p_{k}=\theta$. Then, for $j=1,2,3, \ldots$, we define the following set:

$$
A(j)=\left\{k \in \mathbb{N}: p_{k} \in \mathcal{N}_{\theta}\left(\frac{1}{j}\right), j \in \mathbb{N}\right\} .
$$

Since $\delta I-\lim p_{k}=\theta$, it follows that $A^{c}(j) \in I$.

Now, for $j=1,2,3, \ldots$, we observe that $A(j) \supset A(j+1)$ and

$$
A(j) \in \mathscr{F} .
$$

We show that, for $k \in A(j), \mathcal{S}-\lim _{k \rightarrow \infty} p_{k}=\theta$. Suppose that, for $k \in A(j),\left(p_{k}\right)$ is not $\mathcal{S}$-convergent to $\theta$. Then, there exists some $i>0$ such that

$$
\left\{k \in \mathbb{N}: p_{k} \notin \mathcal{N}_{\theta}(i)\right\}
$$

for infinitely many terms $p_{k}$. Let

$$
A(i)=\left\{k \in \mathbb{N}: p_{k} \notin \mathcal{N}_{\theta}(i)\right\}
$$

and $i>1 / j, j=1,2,3, \ldots$. Then, we have $A(i) \in I$. Also, $A(j) \subset A(i)$ implies that $A(j) \in I$, which contradicts (42) as $A(j) \in \mathscr{F}$. Hence, $\mathcal{S}-\lim _{k \rightarrow \infty} p_{k}=\theta$.

Converse part is easy and can be omitted.

\section{Ideal Convergence in Probability and in $L^{p}$ Space}

Let $\left(x_{k}\right)$ be a sequence of random variables defined on a probability space $(\Omega, \mathscr{A}, P)$ taking values in a separable normed space $(X,\|\cdot\|)$, where $\|\cdot\|$ is the norm. Then, we say that a sequence $\left(x_{k}\right)$ converges in probability or converges in measure to $\theta$ if for every $\lambda>0$,

$$
\lim _{k} P\left(\left\|x_{k}\right\|>\lambda\right)=0 .
$$

Equivalently, for any $\lambda>0$, there is an integer $n_{0} \in \mathbb{N}$ such that

$$
P\left(\left\|x_{k}\right\|<\lambda\right)>1-\lambda, \quad \forall k \geq n_{0} .
$$

In this case, we write $x_{k} \stackrel{\mathscr{P}}{\longrightarrow} \theta$.

Now, we give the definition of ideal convergence in probability as follows.

Definition 19. A sequence $\left(x_{k}\right)$ of random variables is said to be I-convergent in probability to $\theta$, if, for every $\lambda>0$, the set

$$
\left\{k \in \mathbb{N}: P\left(\left\|x_{k}\right\|<\lambda\right) \leq 1-\lambda\right\} \in I
$$

or

$$
\left\{k \in \mathbb{N}: P\left(\left\|x_{k}\right\|<\lambda\right)>1-\lambda\right\} \in \mathscr{F} .
$$

In this case, we write $x_{k} \stackrel{I(\mathscr{P})}{\longrightarrow} \theta$ or $I(\mathscr{P})-\lim x_{k}=\theta$.

Example 20. Let $\Omega=[0,1]$ and $P$ a Lebesgue measure on $[0,1]$. Define the sequence of random variables $x_{k}=x_{k}(t)$ for $t \in[0,1]$ as follows:

$$
x_{k}(t)= \begin{cases}\ell, & \text { if } t \in\left[0, \frac{1}{k}\right] \\ 0, & \text { otherwise. }\end{cases}
$$

For any $\varepsilon>0$, we have

$$
\begin{aligned}
P\left(\left\|x_{k}-0\right\|>\varepsilon\right) & =P\left(x_{k}(t)=\ell\right)=P\left(t \in\left[0, \frac{1}{k}\right]\right) \\
& =\text { length of the interval }=\frac{1}{k} \longrightarrow 0 .
\end{aligned}
$$

It means that $\left(x_{k}\right)$ is $I$-convergent in probability to zero. That is, $x_{k} \stackrel{I(\mathscr{P})}{\longrightarrow} 0$.

Theorem 21. Let $\left(f_{k}\right)$ be a sequence of (equivalence classes of) $E$-valued random variables. Then, the following are equivalent:

(i) $f_{k} \stackrel{I(\mathscr{P})}{\longrightarrow} \theta_{S}$;

(ii) $v_{f_{k}} \stackrel{\mathscr{W} I}{\longrightarrow} \varepsilon_{0}$;

(iii) $f_{k} \stackrel{I}{\rightarrow} \theta_{S}$ in the ̌̌erstnev space $\left(L^{0}, v, \tau_{W}\right)$.

Proof. By definition, it is clear that (ii) and (iii) are equivalent, and it suffices to establish the equivalence of (i) and (ii).

Let $K(\lambda)=\left\{k \in \mathbb{N}: P\left(\left\|f_{k}\right\|<\lambda\right) \leq 1-\lambda\right\}$. We note that $f_{k} \stackrel{I(\mathscr{P})}{\longrightarrow} \theta_{S}$ if and only if $K(\lambda) \in I$. But $\mathbb{N} \backslash K(\lambda) \in \mathscr{F}$. Therefore, for every $k \in \mathbb{N} \backslash K(\lambda)$, we have $P\left(\left\|f_{k}\right\|<\lambda\right)>1-\lambda$. By (17), implies that $v_{f_{k}}(\lambda)>1-\lambda$. By the property of $\mathcal{S}$ topology, we have

$$
\begin{aligned}
\left\{k \in \mathbb{N}: d_{L}\left(v_{f_{k}}, \varepsilon_{0}\right)<\lambda\right\} & \supseteq\left\{k \in \mathbb{N}: v_{f_{k}}(\lambda)>1-\lambda\right\} \\
& \supseteq\left\{k \in \mathbb{N}: P\left(\left\|f_{k}\right\|<\lambda\right)>1-\lambda\right\} ;
\end{aligned}
$$


that is,

$$
\left\{k \in \mathbb{N}: d_{L}\left(v_{f_{k}}, \varepsilon_{0}\right) \geq \lambda\right\} \subseteq\left\{k \in \mathbb{N}: P\left(\left\|f_{k}\right\|<\lambda\right) \leq 1-\lambda\right\} .
$$

Since $f_{k} \stackrel{I(\mathscr{P})}{\longrightarrow} \theta_{S}$, therefore $\left\{k \in \mathbb{N}: P\left(\left\|f_{k}\right\|<\lambda\right) \leq\right.$ $1-\lambda\} \in I$.

Thus, $\left\{k \in \mathbb{N}: d_{L}\left(\nu_{f_{k}}, \varepsilon_{0}\right) \geq \lambda\right\} \in I$; hence $v_{f_{k}} \stackrel{\mathscr{W} I}{\longrightarrow} \varepsilon_{0}$.

In order to consider ideal convergence in $L^{p}$ with $p \in$ $[1,+\infty)$, the following result connecting the $L^{p}$ norms $\|\cdot\|_{p}$ with the probabilistic norm (17) will be needed (see [38]).

Theorem 22. Let $L^{p}=L^{p}(\Omega, \mathscr{A}, P):=\left\{f \in L^{0}: \int_{\Omega}|f|^{p} d P<\right.$ $+\infty\}$ for $p \in[1,+\infty)$ and $L^{\infty}:=\left\{f \in L^{0}:\|f\|_{\infty}:=\sup |f|<\right.$ $+\infty\}$. If the probabilistic norm $v: L^{0} \rightarrow \Delta^{+}$is defined by

$$
\nu_{f}(\lambda):=P(\{\omega \in \Omega:|f(\omega)|<\lambda\}), \quad \lambda>0,
$$

then for every $f \in L^{p},\|f\|_{p}=\left(\int_{\mathbb{R}_{+}} \lambda^{p} d \nu_{f}(\lambda)\right)^{1 / p}$, and for every $f \in L^{\infty},\|f\|_{\infty}=\sup \left\{t>0: \nu_{f}(t)<1\right\}$.

With the help of Theorem 22, one can characterize ideal convergence in $L^{p}$.

Theorem 23. Let $\left(f_{k}\right)$ be a sequence of (equivalence classes of) E-valued random variables in $L^{p}$. Then, the following statements are equivalent.

If $p \in[1,+\infty)$,

(i) $f_{k} \stackrel{I\left(L^{p}\right)}{\longrightarrow} \theta_{S}$;

(ii) the sequence of the pth moments of the probabilistic norms $\left(v_{f_{k}}\right)$ I-converges to 0.

If $p=+\infty$,

(iii) $f_{k} \stackrel{I\left(L^{\infty}\right)}{\longrightarrow} \theta_{S}$;

(iv) for every $\lambda>0,\left\{k \in \mathbb{N}: \nu_{f_{k}}(\lambda)=1\right\} \in \mathscr{F}$.

Proof. (i) $\Leftrightarrow$ (ii) We note that $f_{k} \stackrel{I\left(L^{p}\right)}{\longrightarrow} \theta_{S} \Leftrightarrow\left\{k \in \mathbb{N}:\left\|f_{k}\right\|_{p} \geq\right.$ $\lambda\} \in I$ for every $\lambda>0$. But

$$
\left\{k \in \mathbb{N}:\left(\int_{\mathbb{R}_{+}} t^{p} d v_{f_{k}}(t)\right)^{1 / p} \geq \lambda\right\}=\left\{k \in \mathbb{N}:\left\|f_{k}\right\|_{p} \geq \lambda\right\} .
$$

Hence,

$$
\left\{k \in \mathbb{N}: \int_{\mathbb{R}_{+}} t^{p} d v_{f_{k}}(t) \geq(\lambda)^{p}\right\} \in I .
$$

(iii) $\Rightarrow$ (iv) Suppose that $f_{k} \stackrel{I\left(L^{\infty}\right)}{\longrightarrow} \theta_{S}$, that is, $\left\|f_{k}\right\|_{\infty} \stackrel{I\left(L^{\infty}\right)}{\longrightarrow}$ 0 , and let $t>0$, then, for every $0<\varepsilon<t$, we have

$$
\begin{gathered}
\left\{k \in \mathbb{N}: \sup \left\{t>0: v_{f_{k}}(t)<1\right\} \geq \varepsilon\right\} \\
=\left\{k \in \mathbb{N}:\left\|f_{k}\right\|_{\infty} \geq \varepsilon\right\} \in I .
\end{gathered}
$$

This implies that

$$
\left\{k \in \mathbb{N}: \sup \left\{t>0: v_{f_{k}}(t)<1\right\}<\varepsilon\right\} \in \mathscr{F} \text {. }
$$

Hence,

$$
\left\{k \in \mathbb{N}: v_{f_{k}}(t) \geq v_{f_{k}}(\varepsilon)=1\right\} \in \mathscr{F} .
$$

(iv) $\Rightarrow$ (iii) For $t>0$, suppose that $\left\{k \in \mathbb{N}: v_{f_{k}}(t)=1\right\} \in$ $\mathscr{F}$, and therefore

$$
\left\{k \in \mathbb{N}:\left\|f_{k}\right\|_{\infty}<t\right\} \in \mathscr{F},
$$

which implies that $\left\|f_{k}\right\|_{\infty} \stackrel{I\left(L^{\infty}\right)}{\longrightarrow} 0$.

\section{Acknowledgments}

The authors gratefully acknowledge the financial support from King Abdulaziz University, Jeddah, Saudi Arabia.

\section{References}

[1] H. Fast, "Sur la convergence statistique," Colloquium Mathematicum, vol. 2, pp. 241-244, 1951.

[2] H. Steinhaus, "Sur la convergence ordinaire et la convergence asymptotique," Colloquium Mathematicum, vol. 2, pp. 73-74, 1951.

[3] J. A. Fridy, "On statistical convergence," Analysis, vol. 5, no. 4, pp. 301-313, 1985.

[4] T. Šalát, "On statistically convergent sequences of real numbers," Mathematica Slovaca, vol. 30, no. 2, pp. 139-150, 1980.

[5] I. J. Maddox, "Statistical convergence in a locally convex space," Mathematical Proceedings of the Cambridge Philosophical Society, vol. 104, no. 1, pp. 141-145, 1988.

[6] S. A. Mohiuddine and M. Aiyub, "Lacunary statistical convergence in random 2-normed spaces," Applied Mathematics \& Information Sciences, vol. 6, no. 3, pp. 581-585, 2012.

[7] S. A. Mohiuddine and M. A. Alghamdi, "Statistical summability through a lacunary sequence in locally solid Riesz spaces," Journal of Inequalities and Applications, vol. 2012, article 225, 2012.

[8] S. A. Mohiuddine, A. Alotaibi, and M. Mursaleen, "Statistical convergence through de la Vallée-Poussin mean in locally solid Riesz spaces," Advances in Difference Equations, vol. 2013, article 66, 2013.

[9] S. A. Mohiuddine, A. Alotaibi, and M. Mursaleen, "Statistical convergence of double sequences in locally solid Riesz spaces," Abstract and Applied Analysis, vol. 2012, Article ID 719729, 9 pages, 2012.

[10] C. Belen and S. A. Mohiuddine, "Generalized weighted statistical convergence and application," Applied Mathematics and Computation, vol. 219, no. 18, pp. 9821-9826, 2013.

[11] P. Kostyrko, T. Šalát, and W. Wilczyński, “I-convergence," Real Analysis Exchange, vol. 26, no. 2, pp. 669-685, 2001.

[12] K. Dems, “On I-Cauchy sequences," Real Analysis Exchange, vol. 30, no. 1, pp. 123-128, 2005.

[13] H. Cakalli and B. Hazarika, "Ideal quasi-Cauchy sequences," Journal of Inequalities and Applications, vol. 2012, article 234, 2012. 
[14] P. Das, P. Kostyrko, W. Wilczyński, and P. Malik, "I and $I^{*}$ convergence of double sequences," Mathematica Slovaca, vol. 58, no. 5, pp. 605-620, 2008.

[15] B. Hazarika and E. Savas, "Some $I$-convergent $\lambda$-summable difference sequence spaces of fuzzy real numbers defined by a sequence of Orlicz functions," Mathematical and Computer Modelling, vol. 54, no. 11-12, pp. 2986-2998, 2011.

[16] M. Mursaleen, S. A. Mohiuddine, and O. H. H. Edely, "On the ideal convergence of double sequences in intuitionistic fuzzy normed spaces," Computers \& Mathematics with Applications, vol. 59, no. 2, pp. 603-611, 2010.

[17] K. Menger, "Statistical metrics," Proceedings of the National Academy of Sciences of the United States of America, vol. 28, pp. 535-537, 1942.

[18] A. N. Šerstnev, "Random normed spaces: problems of completeness," vol. 122, pp. 3-20, 1962.

[19] A. N. Šerstnev, "On the concept of a stochastic normalized space," Doklady Akademii Nauk SSSR, vol. 149, pp. 280-283, 1963.

[20] C. Alsina, B. Schweizer, and A. Sklar, "On the definition of a probabilistic normed space," Aequationes Mathematicae, vol. 46, no. 1-2, pp. 91-98, 1993.

[21] M. Mursaleen and S. A. Mohiuddine, "On ideal convergence in probabilistic normed spaces," Mathematica Slovaca, vol. 62, no. 1, pp. 49-62, 2012.

[22] M. Mursaleen and S. A. Mohiuddine, "On ideal convergence of double sequences in probabilistic normed spaces," Mathematical Reports, vol. 12, no. 62, pp. 359-371, 2010.

[23] M. Mursaleen and A. Alotaibi, "On $I$-convergence in random 2-normed spaces," Mathematica Slovaca, vol. 61, no. 6, pp. 933940, 2011.

[24] S. A. Mohiuddine, A. Alotaibi, and S. M. Alsulami, "Ideal convergence of double sequences in random 2-normed spaces," Advances in Difference Equations, vol. 2012, article 149, 2012.

[25] M. Gürdal and I. Açık, "On I-Cauchy sequences in 2-normed spaces," Mathematical Inequalities \& Applications, vol. 11, no. 2, pp. 349-354, 2008.

[26] B. Hazarika, "On generalized difference ideal convergence in random 2-normed spaces," Filomat, vol. 26, no. 6, pp. 1265-1274, 2012.

[27] V. Kumar and B. L. Guillén, "On ideal convergence of double sequences in probabilistic normed spaces," Acta Mathematica Sinica, vol. 28, no. 8, pp. 1689-1700, 2012.

[28] K. Kumar and V. Kumar, "On the $I$ and $I^{*}$-Cauchy sequences in probabilistic normed spaces," Mathematical Sciences, vol. 2, no. 1, pp. 47-58, 2008.

[29] B. K. Lahiri and P. Das, " $I$ and $I^{*}$-convergence in topological spaces," Mathematica Bohemica, vol. 130, no. 2, pp. 153-160, 2005.

[30] S. A. Mohiuddine, H. Şevli, and M. Cancan, "Statistical convergence in fuzzy 2-normed space," Journal of Computational Analysis and Applications, vol. 12, no. 4, pp. 787-798, 2010.

[31] A. Şahiner, M. Gürdal, S. Saltan, and H. Gunawan, "Ideal convergence in 2-normed spaces," Taiwanese Journal of Mathematics, vol. 11, no. 5, pp. 1477-1484, 2007.

[32] B. Schweizer and A. Sklar, "Statistical metric spaces," Pacific Journal of Mathematics, vol. 10, pp. 313-334, 1960.

[33] U. Yamanci and M. Gürdal, "On lacunary ideal convergence in random 2-normed space," Journal of Mathematics, vol. 2013, Article ID 868457, 8 pages, 2013.
[34] B. Schweizer and A. Sklar, Probabilistic Metric Spaces, NorthHolland Series in Probability and Applied Mathematics, NorthHolland, New York, NY, USA, 1983.

[35] D. A. Sibley, "A metric for weak convergence of distribution functions," The Rocky Mountain Journal of Mathematics, vol. 1, no. 3, pp. 427-430, 1971.

[36] E. P. Klement, R. Mesiar, and E. Pap, Triangular Norms, vol. 8 of Trends in Logic, Kluwer Academic, Dodrecht, The Netherlands, 2000.

[37] C. Alsina, B. Schweizer, and A. Sklar, "Continuity properties of probabilistic norms," Journal of Mathematical Analysis and Applications, vol. 208, no. 2, pp. 446-452, 1997.

[38] B. L. Guillén, J. A. R. Lallena, and C. Sempi, "Some classes of probabilistic normed spaces," Rendiconti di Matematica e delle sue Applicazioni, vol. 17, no. 2, pp. 237-252, 1997. 


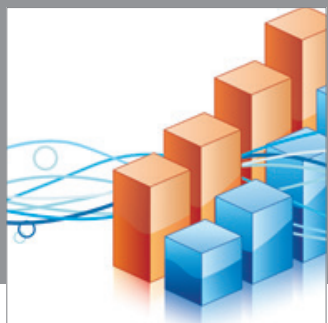

Advances in

Operations Research

mansans

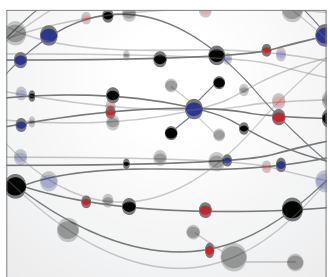

The Scientific World Journal
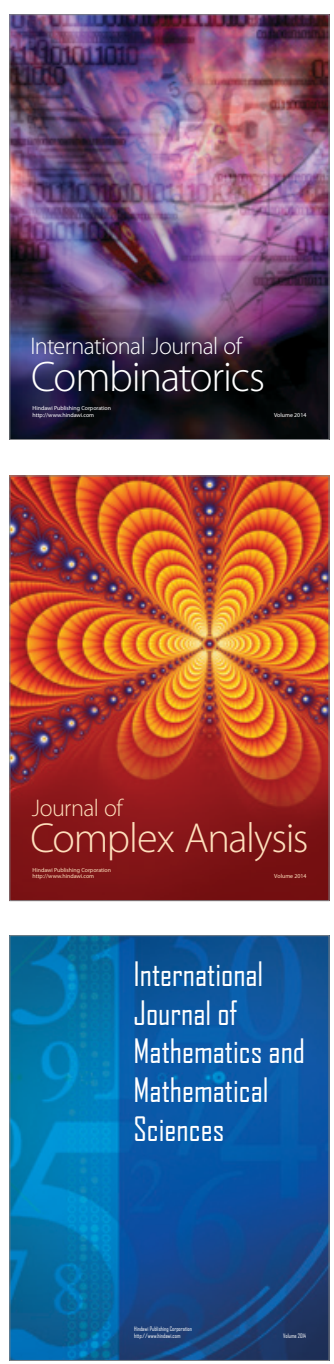
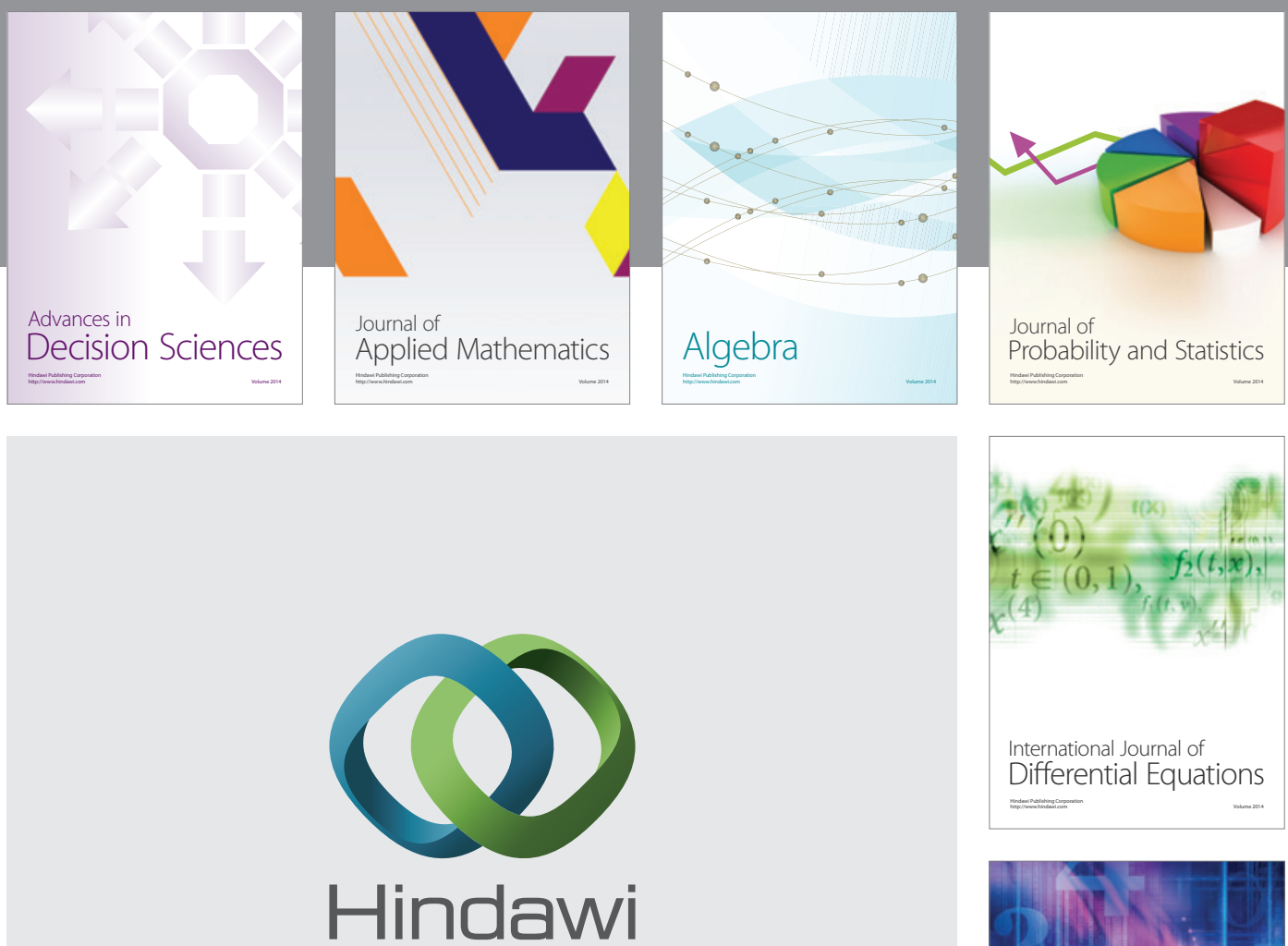

Submit your manuscripts at http://www.hindawi.com
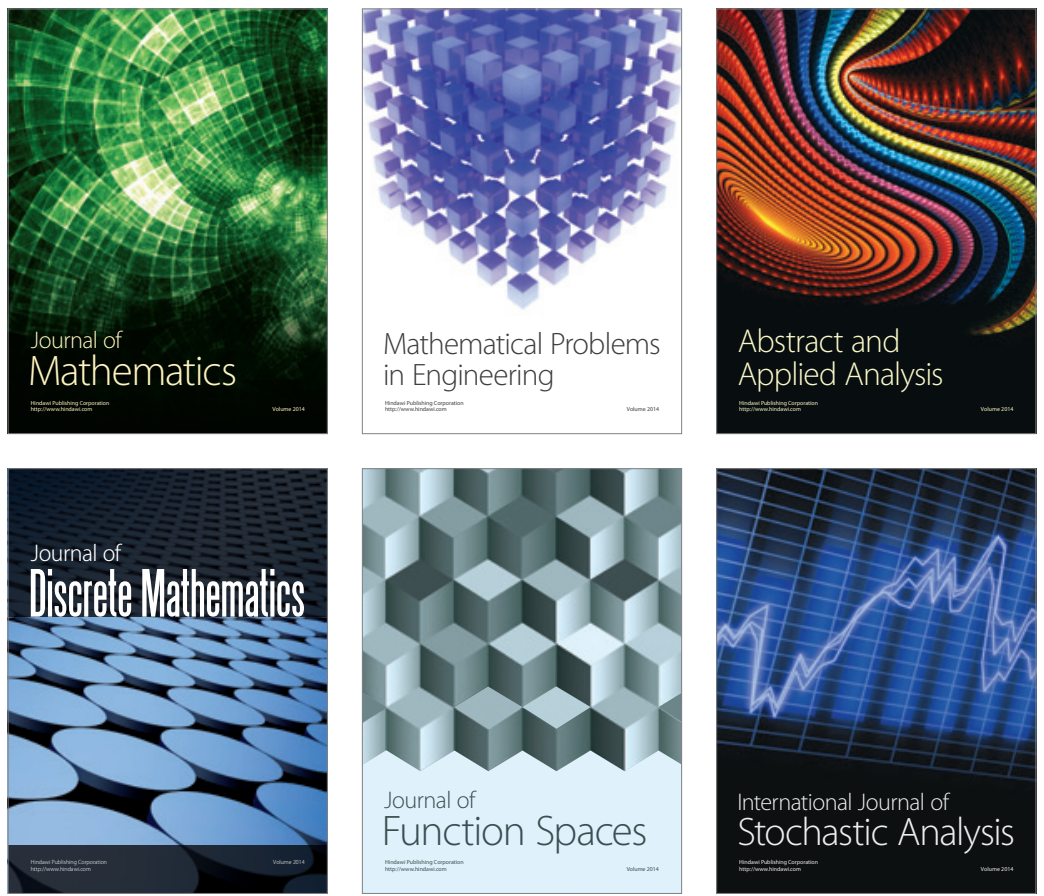

Journal of

Function Spaces

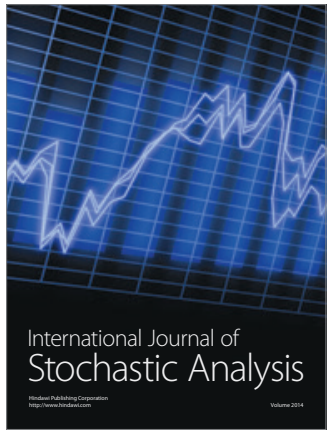

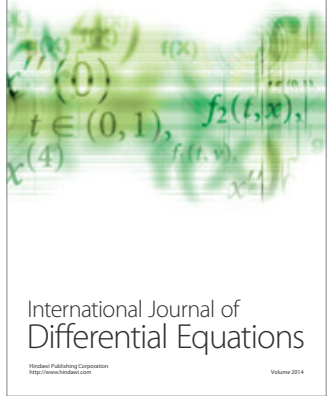
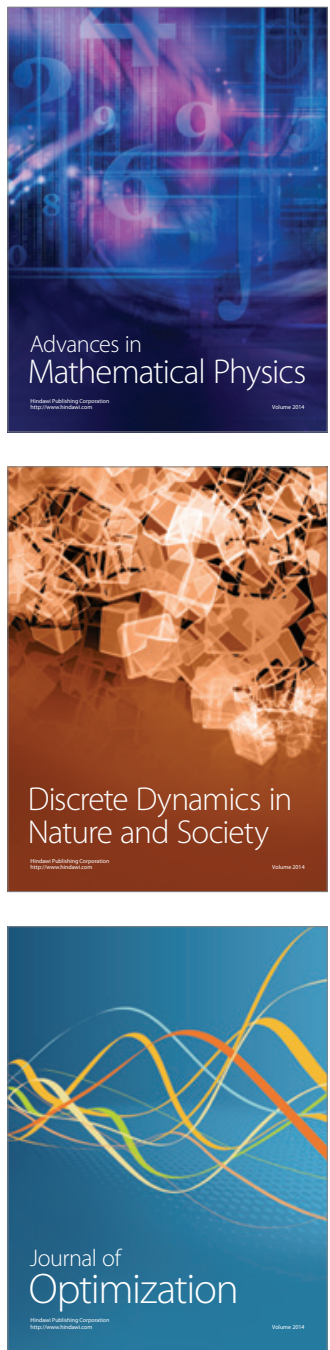